\title{
La necesidad de herramientas para la co-creación del paisaje urbano
}

\author{
The need for a toolkit for urban landscape co-creation
}

A necessidade de ferramentas para a co-criação da paisagem urbana

Hans Intveen Pérez. M.Sc. en Urbanismo.

hintveen@gmail.com

iD https://orcid.org/0000-0001-8953-4137

Universidad de Chile. Ministerio de Vivienda y Urbanismo, Región de Antofagasta, Chile.
Recibido: Julio 25 de 2018 Aceptado: Octubre 06 de 2018 Publicado: Diciembre de 2018

\section{RESUMEN}

Con el objetivo de acercarse a una participación ciudadana oportuna, legible y medible, en el contexto del desarrollo del paisaje urbano en la ciudad latinoamericana, se desarrolla una herramienta para la extracción de información espacial desde la comunidad, a través de patrones de diseño aplicables a la obra urbana. Se sistematiza una experiencia de diseño participativo en la Región de Antofagasta -en el marco del Programa de Recuperación de Barrios del Ministerio de Vivienda y Urbanismo-, demostrando la importancia de acercarse a un co-diseño del paisaje urbano a nivel de barrio, ciudad y territorio.

Palabras clave: participación; co-diseño; ciudadanía.

\begin{abstract}
With the aim of approaching a timely, readable and measurable citizen participation, in the context of the development of the urban landscape in the Latin American city, a tool is developed for the extraction of spatial information from the community, through design patterns applicable to the urban work. A participatory design experience is systematized in the Antofagasta Region -in the framework of the Neighborhood Recovery Program of the Ministry of Housing and Urbanism-, demonstrating the importance of approaching a codesign of the urban landscape at the neighborhood, city and territory.
\end{abstract}

Key words: participation; co-design; citizenship.

\section{RESUMO}

Com o objetivo de abordar uma participação cidadã oportuna, legível e mensurável, no contexto do desenvolvimento da paisagem urbana na cidade latino-americana, é desenvolvida uma ferramenta para a extração de informações espaciais da comunidade, através de padrões de projeto aplicáveis a o trabalho urbano. Uma experiência de design participativo é sistematizada na Região de Antofagasta - no âmbito do Programa de Recuperação de Vizinhança do Ministério da Habitação e Urbanismo-, demonstrando a importância de se abordar um co-design da paisagem urbana no bairro, cidade e região, território.

Palavras-chave: participação; co-design; cidadania. 


\section{Introducción}

\section{Ciudadanía y Participación}

Ciudadanía era un concepto que, originalmente, se tenía reservado a la pertenencia a una ciudad: se era ciudadano de Atenas, de Roma, de París. Solo de manera tardía fue desplazando su significado hacia la pertenencia al EstadoNación. Pero todas las ciudadanías tienen algo en común; se vislumbran ciertos derechos que provienen del hecho de pertenecer a ese territorio. Uno de ellos, quizás el principal, es participar de las decisiones públicas. En la misma línea, se plantea, con razón, que en los proyectos públicos existe una auténtica "oportunidad de producir ciudadanía" (Borja, 1998). Y su adecuada gestión representa, al mismo tiempo, "un test del desarrollo de la misma". En otras palabras: mejores espacios públicos (participativos, vitales, representativos) son un signo de una mejor ciudadanía, y se traducen en una mejor ciudad. Así, una participación auténticamente "ciudadana" no sólo es necesaria para efectos de poder llevar a cabo un diseño, sino que es un insumo fundamental en la futura gobernabilidad urbana. El espacio mismo de la ciudad debe cumplir una función social y alentar la participación, como se puede leer en el documento de Habitat III (ONU, 2017, p.5).

En el contexto chileno, el Programa Quiero mi Barrio (PQMB) del Ministerio de Vivienda y Urbanismo (MINVU) es, en este sentido, un auténtico laboratorio de experiencias urbanas participativas. Se trata de un programa que aborda el desarrollo urbano como una suma de pequeñas dosis (Alexander, 1976), donde el habitante también hace las veces de "diseñador", con una metodología de intervención que ya posee una década de refinamiento y que integra a este habitante, haciéndolo partícipe tanto del proceso de formulación como el diseño y la ejecución. Ahora, pese a haber cumplido una década y ser un programa exitoso como política pública, todavía presenta desafíos que tienen que ver con la complejidad de la realidad misma, y con un estado que se moderniza y plantea desafíos de eficiencia y focalización de las inversiones. Sin embargo, en su calidad de laboratorio de buenas prácticas participativas, es un programa del cual ya se pueden establecer aprendizajes válidos para mejorar los procesos de toma de decisiones en el ámbito urbano. Principalmente, debido a las siguientes razones: incorpora desde el inicio la información y demandas de la gente; resuelve a tiempo los conflictos, explicitando y conciliando distintos intereses; y crea capital social, empoderando a los ciudadanos como líderes de los procesos de desarrollo barrial.

Es, precisamente, este aprendizaje producido por la aproximación participativa que tiene el PQMB frente a los desafíos urbanos, que plantean las ciudades actuales en su dinámica barrial, el que evidencia un quiebre metodológico al momento de llegar a un nivel de detalle correspondiente al de proyecto urbano. Especialmente, cuando se trata de proyectos que no se encuentran al alero del programa. Este quiebre se produce debido a que estos proyectos son desarrollados - casi la totalidad de las veces- por empresas consultoras, ya que los equipos profesionales de los gobiernos locales ${ }^{1}$ resultan insuficientes para llegar a este nivel de detalle proyectual. Este proceso de privatización de la responsabilidad de diseño es una tendencia que, más allá de la opinión que se pueda tener en términos de pertinencia, se ha impuesto en las últimas décadas y, asimismo, se ha expandido en todos los ámbitos del Estado.

Entonces, es precisamente en este punto donde se evidencia una problemática relevante que lleva al siguiente cuestionamiento: ¿Cuál es la capacidad que pueden tener estas empresas de lograr una adecuada inserción en el territorio? Esto es particularmente relevante en casos en los cuales las consultoras - por una lógica de mercado y especialización- resultan ser oficinas de alcance nacional que no tienen una relación cercana con el territorio.

De esta forma, uno de los principales objetivos de esta investigación es encaminarse hacia una adecuada "extracción" de la información y su correspondiente traducción a parámetros claros que orienten el diseño. En otras palabras, sistematizar un instrumento que sirva para una realidad particular que se genera en las consultorías de diseño, que sea capaz, en pocas jornadas, de extraer información precisa sobre las preferencias que tienen los habitantes sobre los espacios comunes. La hipótesis de trabajo en este estudio es relativamente sencilla en su formulación, pero compleja en su puesta en práctica: Una adecuada extracción de la información que la ciudadanía ya posee, hará posible generar patrones de diseño que serán utilizados con eficiencia en el posterior diseño del espacio público. Esto, en el entendido que estos patrones serían plenamente generados por los habitantes. Bajo este esquema, la labor del proceso de diseño participativo sería, entonces, consensuar estos patrones y que todo este proceso pueda desembocar en un formato práctico, para que puedan ser, posteriormente, traducidos en espacios y situaciones urbanas concretas. De esta forma, se facilita el objetivo de que los habitantes sean cocreadores de paisajes basados en patrones propios, alimentados por un auténtico sentido de comunidad, cuestión que se demostró como fundamental en las restauraciones paisajísticas posteriores al terremoto de 2010 (Intveen, 2016, p.36).

Las limitantes del diseño participativo en el escenario actual En Chile, la planificación y gestión de los asentamientos humanos es responsabilidad del MINVU, donde se genera la legislación, las políticas e instrumentos técnicos de planificación que orientan el desarrollo urbano. Si bien, en los últimos años se ha incorporado la participación ciudadana, tanto desde los Instrumentos de Planificación a nivel Territorial (IPT) como en los proyectos de diseño urbano a toda escala, una de las debilidades del sistema es el hecho de que -en la práctica- el trabajo de desarrollo queda a cargo de consultorías privadas, donde los organismos estatales funcionan en calidad de revisores y gestores. En las comunas más ricas, debido a la calidad de los equipos profesionales, la presencia estatal es mayor a través del gobierno local. En contraste, en territorios más vulnerables, donde los equipos profesionales son mínimos, la presencia privada es mayor, ya que, prácticamente, no existe la posibilidad de abordar diseños propios y buena parte de los proyectos provienen de fondos sectoriales de nivel central. Irónicamente, a mayor cantidad de recursos, más Estado.

Ahora, aún en el contexto del PQMB, que de todas formas es una situación territorial excepcional, el desarrollo de estas consultorías de diseño urbano se planifica -conceptualmente hablando- en tres etapas ${ }^{2}$ : una primera etapa, donde se realizan los estudios de base y se define un anteproyecto; una segunda etapa, donde se define por completo el proyecto de arquitectura y el de ingeniería a nivel conceptual; una tercera etapa, donde se abordan los proyectos de especialidades. En cada una de estas etapas, se pueden apreciar ciertas patologías del proceso participativo.

Así, esta primera etapa, pese a que desde el MINVU ya se ha planteado la obligación - vía ley- de que la imagen objetivo de un Plan Regulador Comunal se exprese a la comunidad en un lenguaje "claro y simple" (MINVU, 2018)3 ${ }^{3}$, uno de los errores básicos de los procesos participativos (a nivel proyectual) es que de todas formas suelen empezar sin asumir las conclusiones y trabajo previo como parte del mismo proceso que se encuentra 
en desarrollo. Esto implica que, en muchos talleres, se llega a conclusiones que ya se tenían resueltas por la comunidad, duplicando trabajo y en ocasiones confundiendo a la misma. Al mismo tiempo, algunos de los problemas más frecuentes que se producen tienen que ver con cuestiones más bien sencillas que se relacionan con la sensibilidad con la cual se aborda el diseño urbano. El lenguaje de las jornadas participativas, desde las exposiciones a las encuestas -e incluso el material didácticono siempre es el más adecuado al contexto social. Si bien es cierto en los últimos años se ha hecho más frecuente el uso de simulaciones tridimensionales, todavía prima la planimetría técnica árida y poco amable. En este sentido, será clave cómo se implemente la nueva ley en relación a la transparencia y legibilidad de estos procesos (MINVU, 2018). Además, las maquetas tridimensionales de mejor factura usualmente se reservan para las etapas finales, donde la participación deliberativa es casi nula. Esto, sin considerar, además, que las representaciones tipo render suelen abusar de la tecnología, al mostrar el proyecto en un instante idílico que rara vez se da en la realidad.

Pero, quizás, la dinámica más perjudicial del comienzo es que los datos se levantan a la medida de la conveniencia del diseñador. En muchos casos, los requerimientos de los vecinos no se "espacializan" de manera rigurosa, es decir, se circunscriben a conceptos genéricos sin ubicación precisa, dejando demasiado abierto el espectro de diseño. Básicamente, se acomodan a cualquier propuesta.

Luego, en la segunda etapa, uno de los problemas principales es que se pretende diseñar el $100 \%$ del espacio, haciendo gala de un "Horror Vacui" de características barrocas que, en muchas oportunidades, es fomentado entusiastamente por los organismos del Estado. De esta forma, no siempre se tiene en cuenta el incorporar la iniciativa ciudadana en temas técnicos de relevancia (revestimientos, elección cromática, memoria, etc.), que podrían llenar vacíos de diseño cuidadosamente planificados. En esta instancia, el objetivo principal del proceso participativo debiera ser discutir cómo el proyecto se enlaza dentro de un conjunto mayor que es la ciudad, integrándose con otras iniciativas comunales. Esto, rara vez sucede, ya que las iniciativas suelen verse como esfuerzos aislados para solucionar problemas puntuales. En este sentido, los consultores suelen potenciar este punto de vista individualista (o, en el mejor de los casos, sectorialista), debido a que integración es sinónimo de complejidad y, por consiguiente, mayores tiempos de diseño.

Por último, la tercera etapa se sigue tratando como un momento participativo subvalorado, donde la mayor parte de las veces la calidad de la participación baja ostensiblemente, dejándola a un nivel informativo donde "ya está todo resuelto". Se asume que en esta etapa el aporte vecinal es menor, ya que en estos momentos la consultoría se encuentra abocada al desarrollo de especialidades. La verdad, es una etapa donde se debe resolver lo principal: cómo se pretende ocupar el espacio. Rara vez - salvo que exista un programa gubernamental que apoye en este sentido- queda zanjada formalmente la modalidad en la cual se gestionarán los espacios propuestos, cuestión que es, en definitiva, clave para la sostenibilidad de los proyectos que incorporan paisaje urbano. ¿Cuánto cuesta?, ¿Quién queda a cargo?, ¿Cómo se debe cuidar y proteger este nuevo trozo de paisaje urbano? Esas son las preguntas principales que debieran guiar esta última etapa, y que, rara vez, se alcanzan a responder con realismo.

A partir de este contexto, se pueden establecer al menos tres conclusiones básicas. En primer lugar, existe una rigidez procedimental inherente a la estructura de etapas. Es decir, el avance de un contrato de diseño lo van fijando los aspectos técnicos antes que los sociales, dentro de un calendario que es ajustado, poco flexible y secuencial. Así, las fechas de las jornadas de participación se supeditan al calendario de entregas, lo cual no permite incorporar muchas de las opiniones de los vecinos. En segundo lugar, los procesos evidencian problemas de comunicación. Existe una tendencia de los vecinos a no cuestionar demasiado las propuestas que son difíciles de entender, lo cual genera que los cuestionamientos reales vengan en las etapas de ejecución (cuando realmente se logra entender la propuesta). Por último, la Información suele ser de mala calidad. En la mayor parte de los territorios, los proyectos de diseño urbano parten sobre una auténtica tabula rasa. Incluso en los casos en los cuales se ha desarrollado algún tipo de proceso participativo a nivel barrial (como el caso del PQMB), lo usual es que se entregue un perfil que no es auténticamente útil, o bien no expresa una imagen objetivo clara, ni menos se traduce en un programa de recintos o espacios concretos. En definitiva, no se cuenta con los instrumentos necesarios para que, con posterioridad, se pueda cobrar la palabra al equipo consultor $y$, en algunos casos, no existen instrumentos y/o voluntad para ello, ya que retrasa el proceso. Esto lleva a que las oficinas de diseño planifiquen sus propios procesos particip ativos y los diseñen ex profeso para validar sus respectivas propuestas.

En resumen, de acuerdo con lo expuesto, existe la necesidad de adaptarse (teóricamente e instrumentalmente) a la compleja realidad que se da en la realidad del proceso de diseño por consultoría, pero cualquier adaptación deberá hacerse cargo de esta complejidad. Es decir, hay que considerar que existe una estructura de etapas (lineal y secuencial) que no se adapta a un esquema participativo, que necesariamente se da en múltiples instancias en paralelo. Luego, hay que considerar la dificultad práctica de la lejanía física del consultor con respecto al territorio, ya que no se le exigen profesionales permanentes en terreno. Por último, asumiendo la institucionalidad propia del país, se debe considerar que, necesariamente, habrá un cambio de actores en las distintas etapas de las iniciativas de desarrollo urbano (formulación, diseño, ejecución) con la consiguiente dispersión de responsabilidades y roles frente a la comunidad.

Solamente se logrará avanzar en el involucramiento de la comunidad, en la medida en que se aborden instrumentos de participación que sepan lidiar con estas complejidades. En concreto, que no estén diseñados para situaciones ideales que no se dan en la praxis actual.

\section{La participación y la co-creación: Caso Chile}

En el ámbito del desarrollo urbano, la indiscutible tendencia en la última década ha sido la incorporación del componente de participación ciudadana; a todo nivel, desde los planes reguladores hasta los proyectos urbanos. De hecho, en Chile ya se encuentra publicada $-y$ pronta a promulgar - la Ley $N^{\circ} 21.078^{4}$, que establece como obligatorio a los Instrumentos de Planificación Territorial (IPT) contar con una Imagen Objetivo, generada con el componente de participación ciudadana. Esta ley se hace cargo de lo que ya se puede calificar como un "cambio cultural en la práctica del urbanismo" (MINVU, 2017), orientado a dar un mayor protagonismo a las comunidades organizadas, que, en muchos casos, ya se encuentran en una dinámica de autoconstrucción del paisaje, particularmente en ciudades intermedias de crecimiento acelerado.

De hecho, el realizar un diseño basado en los patrones ciudadanos es algo que viene ganando tracción en Latinoamérica. Por ejemplo, el programa favela-barrio, en Brasil, se ha basado en 
una aproximación cuyo concepto no es sacar una solución de un catálogo de soluciones urbanas estándar, sino acompañar a la comunidad en el diseño de alternativas, llevar la ciudad a los ciudadanos (Magalhães, 1999). Una de las características fundamentales de este programa era, precisamente, instalarse mediante un "Puesto de Orientación Urbanística y Social" en el barrio mismo, similar a lo que sucede con el PQMB y su equipo municipal.

Por otra parte, en Colombia se han abordado programas de mejoramiento integral de barrio que comparten esta misma visión, planteando objetivos ambiciosos que hablan de formular mecanismos que promuevan la "recuperación, tutela y sostenibilidad del Espacio Público existente y el desarrollo de nuevos proyectos de generación del mismo" (MAVDT, 2005, pp.13), y que se han concretado en metodologías participativas concretas. Asimismo, en Perú, el programa de Mejoramiento Integral de Barrios, creado el 2012, mediante Decreto Presidencial, ya expresa en su Punto 2.2 que existirá un "acompañamiento social" que tendrá como objetivo la "participación, empoderamiento y emprendimiento de la comunidad asentada en los barrios urbano-marginales".

En todos estos casos, el denominador común es considerar que el problema urbano no es netamente una cuestión de carácter físico, es un problema socio urbano: hay que hacer ciudadanía en esos lugares.

Además, como se puede apreciar en el análisis que realizan en Colombia (MAVDT, 2005, pp.17-18), los problemas son básicamente los mismos en todos estos países: desarrollo sin planificación, vacíos legales, crecimiento acelerado, incumplimiento de las normas, apatía, falta de autogestión y, por último, una deficiencia en las metodologías empleadas.

Precisamente, para suplir este vacío, en Chile la participación se ha transformado en ley (Ley 20.500, 2011), reconociendo a los habitantes el derecho a "participar en sus políticas, planes y programas", aunque probablemente su redacción es todavía tímida en relación con lo que se requiere en el espacio público. Algo más elocuente es la Política Nacional de Desarrollo Urbano, promulgada el año 2014, donde se sostiene, por ejemplo, que "las decisiones deben ser tomadas con el concurso de la ciudadanía, sobre la base de procesos participativos formales y organizados, en todas las escalas" (MINVU; 2014, p.16). En parte, por este sustento normativo como por la tendencia natural de los últimos años, las licitaciones de diseño en la actualidad incorporan requerimientos cada vez más específicos a las jornadas participativas. Ya no basta cumplir con dos o tres instancias de carácter informativo, como era común en la década pasada. En la actualidad hay que presentar una metodología, validar un "Plan de Participación Ciudadana" adecuado al proyecto, involucrar a la comunidad y a los actores locales, como se puede apreciar en las normativas de participación ciudadana que los Servicios de Vivienda y Urbanización (SERVIU) de cada región han promulgado. ${ }^{5}$

Ahora, aún a pesar de todos estos avances, estas buenas intenciones rara vez suelen darse en la realidad. Por esta razón, el MINVU ha creado programas como el "Quiero mi Barrio", para dar sostenibilidad social a las intervenciones en el territorio. Eso sí, es necesario reconocer que, en términos prácticos, esta aproximación todavía se da el ámbito marginal de la presencia territorial del PQMB, pero no ha permeado a todos los proyectos de inversión pública. La mayor parte de los proyectos se trabajan bajo un paradigma netamente pragmático, que se enfoca en la ejecución presupuestaria antes que la pertinencia, y donde no se ha realizado un trabajo previo con la comunidad. En el mejor de los casos, se da ese tipo de participación que viene netamente desde la institucionalidad, y que se ubica en la escala de Arnstein, en lo que se podría llamar como "fachada de participación" (Villarroel, 2014, p.101): cumplir una etapa para lograr un producto y de esta forma validar un diseño.

Aquí es donde ingresa la empresa consultora, que realiza los diseños de arquitectura e ingeniería y coordina jornadas de diseño participativo orientadas a la definición del proyecto, a quien por razones prácticas le acomoda este tipo de participación como "fachada". Así, la pertinencia misma del proceso se pone en juego en este punto, debido a que existe una mayor dispersión en las responsabilidades al incluir a un actor privado que, además, siempre será reacio a incorporar el concepto de co-creación que se plantea en muchos de los estudios actuales y que implica, forzosamente, perder algo de control sobre el diseño.

Es, precisamente, en este momento donde -si se quiere proponer algo que mejore este esquema- conviene repasar algunos conceptos de participación ciudadana que han marcado pauta en la historia de la disciplina, y que se enlazan tanto con la tradición como con la vanguardia. En ese sentido, una de las más interesantes aproximaciones -debido a la abundancia de similitudes que ofrece con el PQMB, que hemos tomado como referencia- es lo planteado por un autor - a estas alturas clásico en lo referente a urbanismo- como Christopher Alexander.

Esta similitud resulta pertinente de destacar debido a que existe una tendencia en otros programas del ministerio (y de otros ministerios, e incluso programas similares de otros países), a asimilar aspectos de la metodología del PQMB, que ya se encuentra probada en el territorio, y que se estructura con base en un proceso participativo comunitario que conduce hacia las siguientes acciones: 1) La creación de una organización social funcional que guiará todas las transformaciones del entorno y asesorará el diagnóstico de la situación del barrio, el Consejo Vecinal de Desarrollo (CVD); 2) El desarrollo, en conjunto con la comunidad de un Plan Maestro de Recuperación de Barrios (PMRB), que propenda a generar una intervención integral y sostenible en el tiempo; 3) La generación de un compromiso de trabajo, que se traduce en un Contrato de Barrio, documento que tiene como actores al gobierno local, la sociedad civil (a través del CVD) y el gobierno central (a través del ministerio).

Así, de manera muy similar a este esquema, el arquitecto Christopher Alexander, en su libro "Urbanismo y Participación" (Alexander, 1976), ejemplifica un proceso de diseño participativo para la comunidad de alumnos, académicos y funcionarios de la Universidad de Oregon, donde propone otorgar un protagonismo inédito al usuario. En este estudio experimental, Alexander sistematiza una serie de criterios que, pese a su antigüedad, cobran mayor vigencia que nunca en una sociedad actual que exige estar involucrada en la gestión de su propio paisaje -así como en la significación de su entorno-y que se asemejan a algunos de los criterios e instrumentos usados por el PQMB.

Tanto lo propuesto por el PQMB como por el método de Alexander, se encaminan al concepto de co-creación, donde la comunidad actúa en un nivel paralelo al diseño "técnico" y se enlaza, además, con las políticas y el estado del arte en la materia. Los principios de crecer a "pequeñas dosis"y "diagnosis" se asemejan bastante al concepto de urbanismo a escala humana, que el Gehl Institute ha propuesto en diversas partes 
del orbe. En el caso chileno, su metodología (que se tradujo en un didáctico manual) propone seis pasos para transformar el espacio público: investigar, envisionar, estrategizar, diseñar, implementar y evaluar (MINVU, 2017, p.71-83).

Tabla 1. Tabla Comparativa entre los Criterios de Alexander y el PQMB.

\begin{tabular}{cc}
\hline $\begin{array}{c}\text { Según C. Alexander } \\
\text { (Alexander, 1976) }\end{array}$ & $\begin{array}{c}\text { Relación con la metodología } \\
\text { del PQMB }\end{array}$ \\
\hline
\end{tabular}

Principio de Orden Orgánico: La Pe realiza un Plan Maestro con planificación y la construcción deben la comunidad, donde pequeñas ser guiadas por un proceso que permita intervenciones de "acupuntura urbana" al todo emerger gradualmente a partir se agrupan para lograr un efecto mayor. de actos locales.

Principio de Participación: Todas las decisiones sobre lo que se ha de construir y sobre cómo se ha de construir deben estar en manos de los usuarios.

os habitantes del barrio deciden qué ideas de proyecto se llevan a cabo, asesorados por un equipo técnico, lo cual se traduce en un Contrato de Barrio, que se firma entre la comunidad, el gobierno local y el Ministerio.

Principio del Crecimiento a Pequeñas Dosis: La construcción emprendida Los proyectos tienen una escala dentro de cada etapa presupuestaria netamente barrial, y se plantean con el debe mesurarse, tendiendo a proyectos objetivo de enlazar con proyectos ya debe mesurarse, tendiendo a proyectos existentes o en proceso de ejecución.

Principio de los Patrones: La construcción y el diseño deben guiarse a través de una colección de principios de planificación, comunitariamente de planificacion, comunita

Se instala un equipo profesiona intervenir, de tal forma que sea el vehículo para canalizar los aportes ciudadanos. Se sistematizan los "modos de habitar" del sector.

Principio de Diagnosis: El bienestar

del todo debe protegerse con un Se realiza un trabajo con la comunidad diagnóstico anual, expresando con para generar competencias que detalle qué espacios están vivos y qué propensdan a mantener y sostener las espacios están muertos, en cualquier obras que se realizan, que se traducen en momento dado de la historia de la un Manual de Acción.

comunidad.

\section{Principio de Coordinación:}

Finalmente, la lenta emergencia de Se dejan instaladas las coordinaciones un orden orgánico en el todo debe entre la comunidad y los organismos estar garantizada por un proceso de del Estado, para lograr el financiamiento financiación que canalice la corriente de una Agenda Futura del barrio en de proyectos individuales prevista por cuestión.

los usuarios.

Fuente: Elaboración propia con base en Alexander (1976)

Pero, para que todos estos principios se puedan implementar en la realidad, es necesario algo más que simplemente "escuchar a las personas":I es necesario diseñar con las personas, para lo cual se necesita tomar los siguientes resguardos:

Que la participación sea oportuna. Las jornadas de participación deben ser en un calendario coordinado con las reuniones técnicas, de tal forma que las decisiones técnicas relativas a factibilidad o gestión puedan ser -idealmentecomunicadas a los vecinos en el momento adecuado. Esto permite interacción real con el habitante y que los requerimientos puedan ser integrados a las decisiones y al desarrollo del proyecto. Asimismo, es necesario resguardar que siempre debe existir un lapso de tiempo adecuado entre las observaciones ciudadanas que permita que la empresa consultora las pueda incorporar con tiempo a la propuesta. No sirve una reunión participativa a dos o tres días de una entrega de proyecto, por ejemplo. En lo posible las observaciones ciudadanas, una vez sistematizadas, debieran estar integradas al pliego de observaciones que entrega la unidad técnica que lleva el proyecto.
Que la participación sea comprensible. El proceso en su conjunto debe tener un lenguaje comprensible por el habitante. Esto es estrictamente necesario para que el proceso tenga un seguimiento adecuado por parte de las organizaciones sociales. No es posible el entendimiento si no hay transparencia, y la transparencia no puede ser validada sin que los actores sociales efectivamente entiendan el proceso. Esto debe aplicarse en todas las etapas y a todo nivel, ya que incluso los actores políticos locales, en muchos casos, quedan al margen debido a una falta de entendimiento.

Que la participación sea medible. Los resultados del proceso participativo deben arrojar criterios que se puedan medir, de tal forma que el habitante -o la autoridad local- pueda realizar un seguimiento adecuado y tenga argumentos precisos para determinar si sus aportes fueron considerados, y en qué medida. Se podría afirmar que hay que "medir la vida urbana", como se ha propuesto el Municipio de Copenhagen, por ejemplo, en su documento "Una Metrópolis para las personas", de 2015 (con asesoría también del Gehl Institute).

Estableciendo una herramienta para diseñar con las personas. Entregar herramientas para que el proceso participativo que se da en las consultorías de diseño urbano mejore respecto a cómo se aborda en la actualidad, es una necesidad imperiosa, ya que el camino hacia un sistema de participación óptimo será largo y requerirá cambios estructurales. Así, en el intertanto mientras estos cambios todavía no se producen- es de suma importancia que se implementen criterios e instrumentos sencillos - de aplicación inmediata- que mejoren el escenario actual, sin necesariamente cambiar esta estructura. Es decir, que se adapten a las circunstancias reales en las cuales se generan los diseños.

Ahora, ¿Cuál es esa realidad? Cualquier instrumento para diseño participativo que deberá tener las siguientes características: a) Debe ser muy acotado en los tiempos. (Con base en dos o tres momentos, que podrían darse en una sola gran jornada o en tres jornadas no distanciadas en el tiempo); b) Debe ser atractivo visualmente, pero simple de implementar. (Al alcance de cualquier equipo, ya sea estatal o privado); c) Debe generar un producto concreto (programa arquitectónico); y, por último, d) Debe haber sido puesto en práctica con algún grado de éxito.

Bajo estos parámetros, tomaremos como base un instrumento que se ha aplicado en diversas jornadas participativas en la Región de Antofagasta en el contexto del PQMB, y que está especialmente pensado para el espacio público. Por las características de este instrumento, el momento oportuno del cual se habla en el capítulo anterior es la primera etapa de un estudio. Se trata, en definitiva, de instrumento diseñado para realizar una zonificación participativa con la comunidad, antes de que exista una imagen de proyecto.

Para realizar este instrumento, se utilizó una adaptación libre de la metodología "Livingstone" (MINVU, 2007, p.31-32) que se fundamenta en el contacto directo entre arquitecto y cliente. Se trata de un método en el cual, en ocasiones, se realizan "juegos de rol"; en este caso, el habitante asume el rol de "diseñador". El sentido del instrumento es que el habitante "clarifique" sus ideas y las traduzca a espacios concretos. El rol del monitor del proceso es ayudar a que el vecino logre consensuar un patrón espacial común (patrón síntesis) con el resto de los habitantes del barrio. Para lograr esta participación, al vecino se le ayuda con un set de "patrones base" que corresponden a situaciones espaciales muy generales. 
Tabla 2. Tabla de Patrones usada en Jornada Participativa.

$\begin{array}{cccc}\text { Set 1 } & \text { Set 2 } & \text { Set 3 } & \text { Set 4 } \\ \begin{array}{c}\text { Situaciones de } \\ \text { carácter "dinámico" }\end{array} & \begin{array}{c}\text { Espacios } \\ \text { relacionados con } \\ \text { "naturaleza" }\end{array} & \text { Espacios "gregarios" } & \begin{array}{c}\text { Espacios relativos a } \\ \text { situaciones "pasivas" }\end{array} \\ \begin{array}{c}\text { Espacios para } \\ \text { deporte }\end{array} & \text { Espacios de agua } & \text { Actividades masivas } & \text { Cobijo artificial } \\ \begin{array}{c}\text { Espacios de juego } \\ \text { (adultos) }\end{array} & \text { Cobijo natural } & \text { Informaciones } & \text { Espacios de culto } \\ \begin{array}{c}\text { Espacios de juego } \\ \text { (niños) }\end{array} & \text { Espacios verdes } & \begin{array}{c}\text { Espacios de venta- } \\ \text { intercambio }\end{array} & \begin{array}{c}\text { Espacios de } \\ \text { descanso }\end{array}\end{array}$

Fuente: elaboración propia

Este denominado patrón base es el primer eslabón de un proceso que tiene una clara inspiración en la metodología empleada por Alexander, y se plantea como una base de apoyo para llegar a un patrón espacial consensuado entre un grupo de vecinos sobre un paisaje común. La simplicidad del patrón base es lo que garantiza que el lenguaje que se está ocupando sea comprensible.
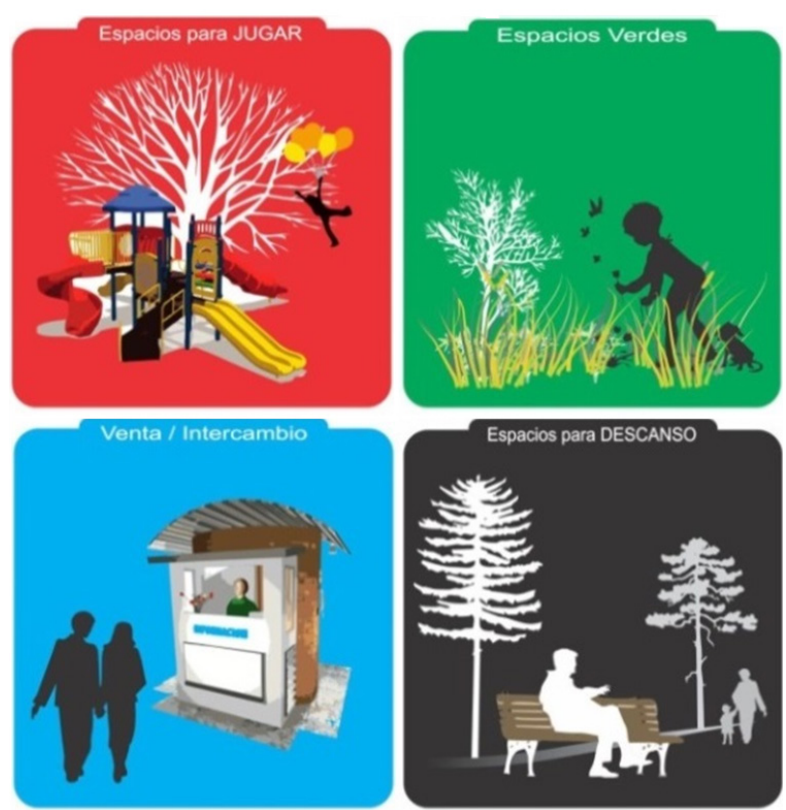

Figura 1. Ejemplos de Patrones y su Representación Gráfica Fuente: Elaboración propia.

El taller se puede plantear como un proceso en una jornada extendida o bien en tres jornadas menores, aunque, en términos de la calidad de la información, mucho más relevante que el número de jornadas es la representatividad de la muestra. Es, en definitiva, lo que le otorga la legitimidad al proceso. La máxima en este sentido es qué estén los que tienen que estar. Es decir, los que toman decisiones sobre el paisaje circundante, pero también quienes ocupan los espacios. En este sentido, la mayor parte de las veces ha sido necesario repetir el taller varias veces, e incluso emplear el sitio mismo donde se va a intervenir como lugar de reunión, de tal forma de poder convocar a quienes no suelen asistir a asambleas convencionales.
Este viaje conceptual hacia un patrón más definido será una discusión que se dará en 3 niveles, que caminarán desde conceptos generales hasta soluciones concretas de diseño.

Tabla 3. Esquema de los Tres Momentos del Instrumento de Diseño Participativo
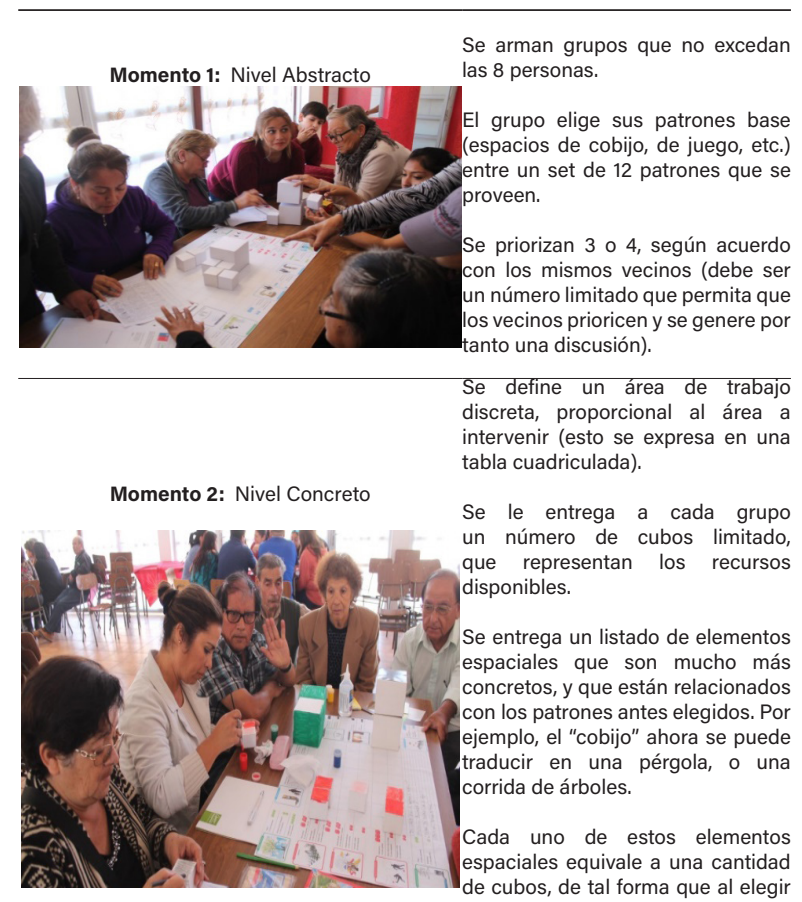
cuadriculada)

Se le entrega a cada grupo un número de cubos limitado, que representan los recursos disponibles.

Se entrega un listado de elementos espaciales que son mucho más concretos, y que están relacionados con los patrones antes elegidos. Por ejemplo, el "cobijo" ahora se puede traducir en una pérgola, o una corrida de árboles.

Cada uno de estos elementos espaciales equivale a una cantidad de cubos, de tal forma que al elegir alguno de estos espacios estarán "gastando" parte de sus recursos (cubos) en un plano espacial.

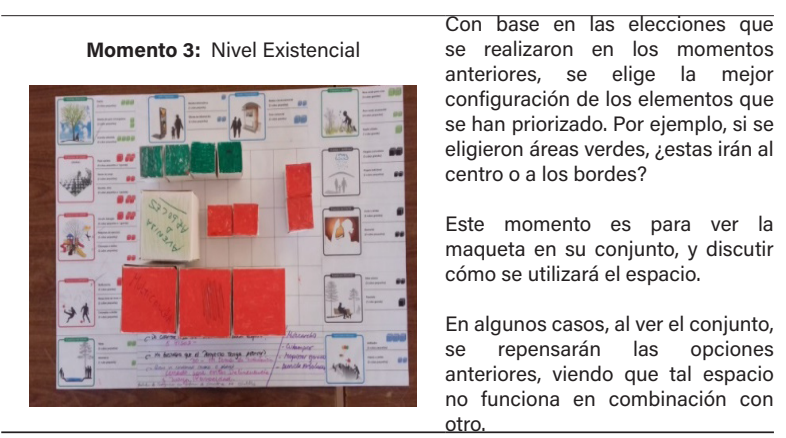

Fuente: Elaboración propia con base en experiencia en proyecto del programa MINVU, de Regeneración de Barrios Vulnerables en Villa "Jorge Alessandri", en Antofagasta, Chile.

La información se entregará a la comunidad (y a la empresa consultora que desarrolla el diseño) de una forma que sea un dato estadístico espacializado, para garantizar que el resultado sea medible. Es decir, un programa arquitectónico respaldado por la opinión de la comunidad.

Este dato se expresará gráficamente (a través del patrón) y estadísticamente (a través de la sistematización de las preferencias), logrando este patrón comunitario o patrón síntesis. 


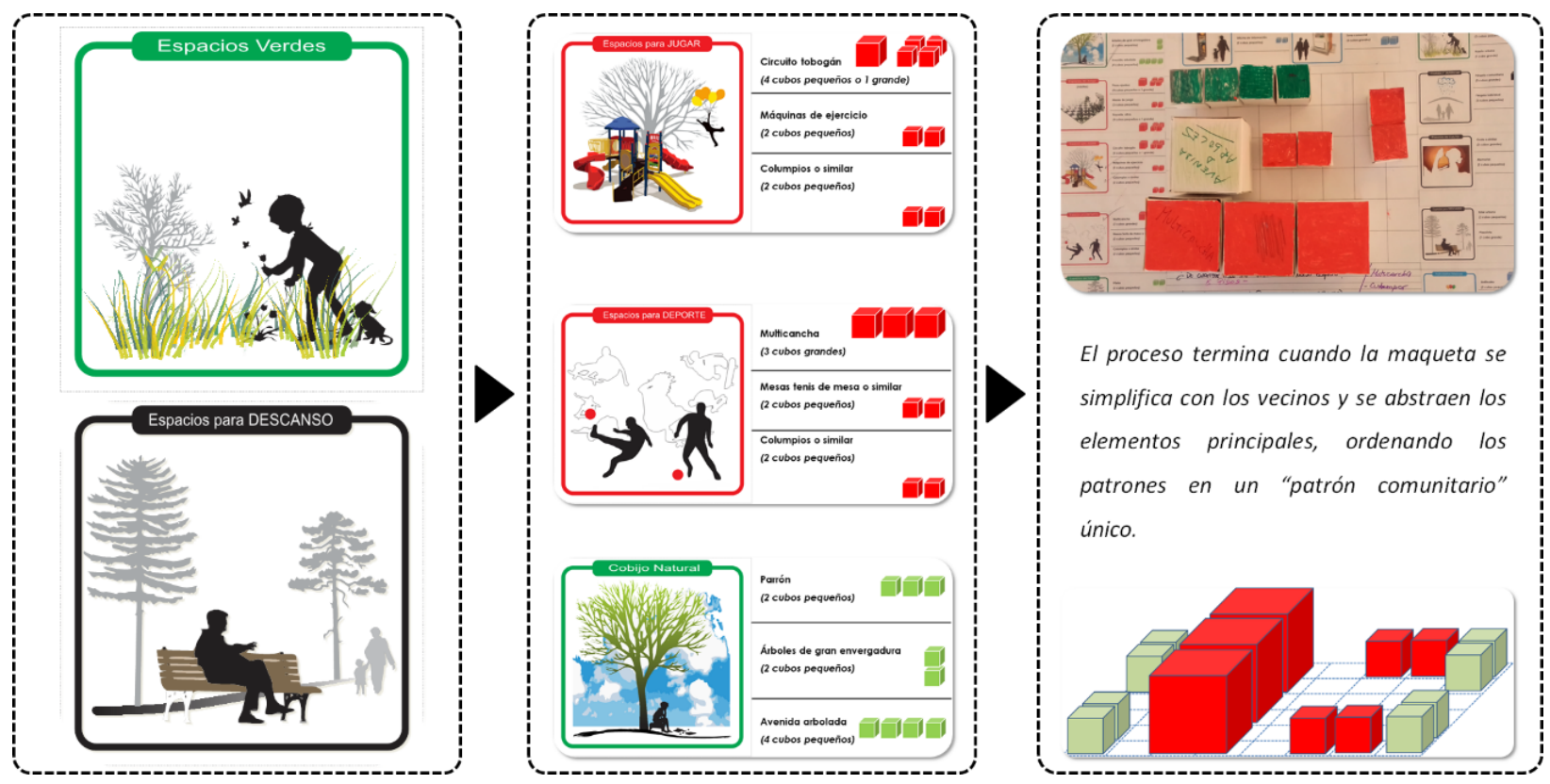

Figura 2. Desarrollo de los patrones en los tres momentos y maqueta resultante.

Fuente: Elaboración propia.

Tabla 4. Resultados en Priorización realizado con vecinos del conjunto "Villa Jorge Alessandri", en Antofagasta.

\begin{tabular}{|c|c|c|}
\hline Primera Prioridad & $\begin{array}{l}\text { Juegos infantiles } \\
32,1 \%\end{array}$ & $\begin{array}{c}\text { Máquinas de ejercicio }(14,3 \%) \\
\text { Circuito tobogán }(10,7 \%) \\
\text { Columpios o similar }(7,1 \%)\end{array}$ \\
\hline Segunda Prioridad & $\begin{array}{c}\text { Espacios verdes } \\
21,4 \%\end{array}$ & $\begin{array}{c}\text { Área verde para estar }(17,9 \%) \\
\text { Huerto Urbano }(7,3 \%) \\
\text { Área verde ornamental }(0 \%)\end{array}$ \\
\hline Tercera Prioridad & $\begin{array}{c}\text { Espacios deportivos } \\
14,3 \%\end{array}$ & $\begin{array}{l}\text { Multicancha }(10,7 \%) \\
\text { Mesas de tenis }(3,6 \%)\end{array}$ \\
\hline
\end{tabular}

Fuente: Elaboración propia en base a ex experiencia en proyecto del programa MINVU de Regeneración de Barrios Vulnerables en Villa "Jorge Alessandri", en Antofagasta, Chile.

\section{Conclusiones}

La conclusión principal del estudio es que un proceso participativo en el ámbito del diseño urbano, en el contexto de una consultoría privada, no es capaz por sí solo de generar una participación ciudadana integral. Tiempos extremadamente acotados, falta de presencia territorial y convocatoria, primacía de las reuniones técnicas en las decisiones relevantes, son algunos de los factores que impiden que este proceso pueda ser abordado de forma eficiente.

Adicionalmente (y como contraste), debe decirse que -en el contexto chileno- lo que se les exige a las consultoras no es realista en relación con las condiciones en las cuales se da el diseño. En algunos casos, desde los organismos estatales, se les piden sofisticadas metodologías que no calzan con la estructura de tiempos en los cuales se diseña. Para mayor abundancia, y relacionado con lo anterior, como el proceso se privatiza por completo, se les otorga una responsabilidad que no corresponde a estas empresas. Uno de los aspectos donde esto se manifiesta con mayor criticidad es en la convocatoria a las reuniones, ya que en muchos casos las oficinas de diseño efectivamente convocan, pero no tienen la misma llegada ciudadana que tiene un gobierno local, el que, a su vez, se desliga de esta responsabilidad debido a que se le ha otorgado a la empresa, a la cual se le paga por ello.

Aun así, lo que sí es posible es mejorar la información que se les entrega a quienes diseñan y mejorar la legitimidad de estos diseños. Para esto, es necesario repensar aspectos del proceso, pero desde una perspectiva realista, pragmática (no excesivamente teórica), mejorando al menos los siguientes aspectos:

Nunca partir de cero. Debe asumirse la información que ya se ha levantado, los planes maestros que se encuentran vigentes en el territorio; en otras palabras, debe entenderse a la consultoría como parte orgánica del proceso, integrando los conceptos que se han generado por el Plan Maestro (en los casos en que exista) y la imagen objetivo que proviene de los Instrumentos de Planificación Territorial. No se puede dejar absolutamente la responsabilidad a la empresa consultora, ya que se trata de una entidad que - por definición- no estará permanentemente en el territorio.

Generar una estructura que ponga al habitante al centro de las decisiones. La estructura de tiempos debe relacionarse con las decisiones que se toman por parte de la ciudadanía, y esto debería traspasarse a las bases de licitación de las obras. En términos contractuales, debería cambiarse la estructura de desarrollo de las licitaciones de diseño, considerando etapas específicas de participación ciudadana independientes, con sus respectivas aprobaciones, estados de pago, etc. Esto incentivaría a las empresas de diseño a tener equipos profesionales dedicados a jornada completa - al menos en estas etapas- y a entregar productos de mejor calidad.

Minimizar esfuerzo al contemplar instancias que generen productos concretos. Para que la participación sea posible, el proceso participativo deber entregar información útil a quienes 
diseñan. Esto es, un programa arquitectónico espacializado, que debe generarse en conjunto con la comunidad. Tomando en cuenta las limitantes de tiempo, el proceso o estructura de trabajo debe contemplar lo justo y necesario para lograr un producto pertinente, sin duplicar labores.

Poder local. En los casos en que exista la capacidad técnica en los gobiernos locales o sectoriales, el proceso participativo debería ser llevado por estas entidades, no por los mismos diseñadores, ya que en términos de diseño terminan siendo juez y parte. Así, en orden a resguardar la objetividad y excelencia del proceso, este no puede transformarse en una instancia de validación de ideas preconcebidas por los mismos diseñadores.

Inclusividad. La pertinencia y mixtura de la convocatoria es clave, mucho más que el número de asistentes. En ese sentido, debe generarse una estrategia (desde el mismo estado) para que la participación sea efectiva, variada y auténticamente inclusiva. No puede dejarse esta responsabilidad enteramente en la empresa.

Generar variables que permitan tener seguimiento. Debe poderse validar el cumplimiento de los objetivos con los cuales se construyó la obra.

En términos más globales, se puede afirmar que el proceso participativo será exitoso si se logra emplazar dentro de una intervención mayor en el territorio, como sucede en el caso del Programa de Recuperación de Barrios (PQMB) u otros de similares características. Pero, en el fondo, lo principal es lograr que las pequeñas dosis de aporte al paisaje comunitario dibujen una idea común, una idea auténticamente ciudadana.

\section{Referencias}

Alexander, C. (1976). Urbanismo y Participación: El caso de la Universidad de Oregon. Barcelona, España: Editorial GG.

Arnstein, S. (1969). A Ladder of Participation. Journal of the American Planning Association, 35:4, 216-224. DOI: 10.1080/01944366908977225

Borja, J. (1998). Ciudadanía y Espacio Público. Revista Ambiente y Desarrollo, 14(3), 13-22. ISSN 0716 - 1476

Comisión Asesora Presidencial (2013). Política Nacional de Desarrollo Urbano. Ciudades sustentables y calidad de vida. Recuperado de: http://politicaurbana.minvu.cl

Intveen, H. (2016). Borde costero resiliente: aprendizaje post-27F para el norte grande de Chile. Procesos Urbanos, 3:24-37. Doi:10.21892/2422085X.265

Magalhães, S. (1999). Cidade Inteira: a política habitacional da cidade do Rio de Janeiro. Prefeitura da Cidade do Rio de Janeiro, Secretaria Municipal de Habitação. Recuperado de http://lam.ibam.org.br

MAVDT Ministerio de Ambiente, Vivienda y Desarrollo Territorial. (2005). Acompañamiento Social aplicado a los Mecanismos de Recuperación y Sostenibilidad del Espacio Público y a la legalización de Asentamientos Precarios de origen ilegal. Bogotá, Colombia: Nuevas Ediciones Ltda.

MINVU (2007). Inventario de Metodologías de Participación Ciudadana en el Desarrollo Urbano. Santiago, Chile: División de Desarrollo Urbano, Monografías y Ensayo MINVU.

MINVU (2015). Norma de Participación Ciudadana. Recuperado de: http://www.minvu.cl

MINVU (2017). La Dimensión Humana en el Espacio Público. Recomendaciones para el Análisis y Diseño. Recuperado de: http://www. minvu.cl/

MINVU (2018). Ley 21.078: Sobre transparencia del mercado del suelo e impuesto al aumento de valor por ampliación del límite urbano. Recuperado de: https://www.bcn.cl

OIDP (2007). Guía para la detección de buenas prácticas en procesos participativos. Recuperado de: http://www.mapama.gob.es

ONU (2017). Nueva Agenda Urbana. Conferencia de las Naciones Unidas sobre la Vivienda y el Desarrollo Urbano Sostenible (Hábitat III), 2016. Recuperado de: http://habitat3.org/wp-content/uploads/NUA-Spanish.pdf

Presidente de la República (2012). Decreto Supremo No4: Crea el Programa de Mejoramiento Integral de Barrios. Recuperado de http:// www3.vivienda.gob.pe/pmib/normatividad.html

Villarroel, C. (2014). Análisis de la participación ciudadana del programa quiero mi barrio: el caso de Valparaíso. Revista labor interdisciplinaria de desarrollo regional, No24, 95-125. ISSN: 0719-526

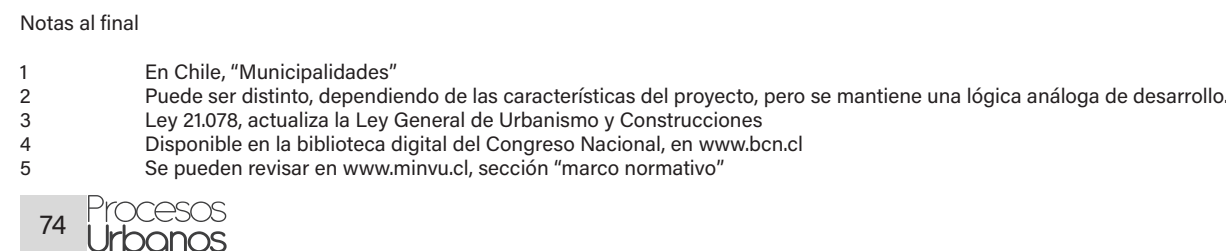

